\title{
Antineoplastons A10 and AS2-1 in the Treatment of Children with Optic Pathway Glioma: Final Report for Protocol BT-23
}

\author{
Stanislaw R. Burzynski ${ }^{1}$, Tomasz J. Janicki ${ }^{1}$, \& Gregory S. Burzynski ${ }^{1}$ \\ ${ }^{1}$ Burzynski Clinic, 9432 Katy Freeway, Houston, Texas 77055, USA \\ Correspondence: Stanislaw R. Burzynski, Burzynski Clinic, 9432 Katy Freeway, Houston, Texas 77055, USA. \\ Tel: 713-335-5697. E-mail: srb@burzynskiclinic.com
}

Received: November 9, 2016

Accepted: December 16, 2016 Online Published: January 6, 2017

doi:10.5539/cco.v6n1p25

URL: http://dx.doi.org/10.5539/cco.v6n1p25

\begin{abstract}
Protocol BT-23, a Phase II study, was performed to determine the efficacy and safety of combination Antineoplaston A10 and Antineoplaston AS2-1 (ANP) in children with an optic pathway glioma (OPG). Sixteen patients (12 males, 4 females) ages 1 to 16 years (median age 4.5 years) were treated. The patients received ANP, by intravenous infusion, six times daily (i.e., every four hours). The length of treatment extended from a minimum of 4.1 weeks to a maximum of 210.0 weeks. The median dosage of A10 was $8.07 \mathrm{~g} / \mathrm{kg} / \mathrm{d}$ while for AS2-1 it was $0.39 \mathrm{~g} / \mathrm{kg} / \mathrm{d}$.

Patients' best response to ANP was determined: $12.5 \%$ of this patient population achieved a complete response, $18.8 \%$ achieved a partial response, and $31.3 \%$ maintained stable disease. The median overall survival was 78.5 months (95\% CI: 37.6-92.3) while median progression-free survival was 45.4 months (95\% CI: 7.1-71.4). ANP was associated with a $12 \%$ incidence of Grade 3 and a $12 \%$ incidence of Grade 4 adverse drug experiences (ADEs). There were no Grade 5 ADEs. Grade 3 ADEs included somnolence and elevated SGOT; Grade 4 ADEs included hypernatremia and hypokalemia. Long-term quality of life was excellent with no chronic toxicity identified. Children with an OPG respond very well to ANP with good efficacy and toxicity profiles.
\end{abstract}

Keywords: Antineoplastons, astrocytoma, glioma, optic pathway glioma, pediatric brain tumors, phase II trial

\section{Introduction}

Optic pathway gliomas (OPG) constitute approximately 6\% of pediatric brain tumors (Jahraus \& Tarbell, 2006; Thiagalingam, Flaherty, Billson \& North, 2004). Typically occurring in children younger than 12 years of age, they primarily involve the optic chiasm and the hypothalamus, but can involve the optic nerve (Edwards \& Cogen, 1994; Janss et al., 1995). Treatment is individualized and prognosis varies. Chiasmatic and hypothalamic tumors are more likely to result in loss of vision and hypothalamic dysfunction, than are gliomas of the optic nerve. However, intra-orbital tumors can also cause loss of vision, as well as proptosis and strabismus. Optic pathway gliomas are the most common type of brain tumor associated with neurofibromatosis 1 (NF-1) and growth of the glioma in some NF-1 patients is so slow that treatment is usually not recommended (Pollack \& Mulvihill, 1996; Tow, Chandela, Miller \& Avellino, 2003). OPGs that occur before the age of one year frequently show a more aggressive growth pattern (Opocher, Kremer, Da Dalt, van de Wetering, Viscardi, Caron \& Perilongo, 2006). Children and adolescents with OPG are generally not candidates for curative surgery. However, when the tumor has a prominent exophytic component, a subtotal resection can be accomplished (Wisoff, Abbott \& Epstein, 1990). Following such a resection, progression-free intervals of greater than 2 years have been reported (Wisoff et al., 1990).

Radiation therapy (RT) is generally avoided in children younger than 3 years of age to prevent subsequent cognitive dysfunction. It is infrequently utilized in the therapy of OPG because of its neurotoxicity. Radiation necrosis, cranial neuropathy, and endocrine dysfunction, including growth hormone deficiency, thyroid dysregulation, and gonadotropin deficiency, are delayed toxicities that can develop in children and adolescents following radiation therapy for OPGs (Burzynski, 2006). Such therapy also predisposes patients to the development of a second primary cancer. "Gamma Knife" therapy, intensity modulated radiation therapy (IMRT), and brachytherapy are being studied as alternatives to standard external beam radiation therapy and offer hope that the incidence of acute and delayed toxicity can be significantly reduced. 
Due to concerns relating to acute and delayed toxicity after radiation therapy, a number of chemotherapeutic regimens have been used in the treatment of OPGs in children and adolescents (Burzynski, 2006). While complete responses (CR) to chemotherapy are unusual, partial response (PR) rates can be substantial (Laithier et al., 2003). Such responses can delay the need for RT (Laithier et al., 2003; Gururangan et al., 2002; Gnekow, Kortmann, Pietsch \& Emser, 2004; Gururangan, et al., 2007). The most widely used chemotherapeutic regimen for the treatment of progressive, low-grade gliomas in children and adolescents is a combination of carboplatin and vincristine (Gururangan et al., 2002; Gnekow et al., 2004). Multiagent platinum-based regimens and nitrosourea-based regimens are also employed (Opocher et al., 2006; Laithier et al., 2003). Temozolomide (TMZ) is reported to be successful in stabilizing disease in 50\% of pediatric patients treated (PR in $12 \%$ and stable disease (SD) in 38\%) (Gururangan et al., 2002). However, the long-term results with TMZ are not as favorable as those obtained using carboplatin containing regimens. There is no doubt that chemotherapy has been successful in stabilizing the disease. Unfortunately, chemotherapy has not cured the disease nor provided a high quality of life for the great majority of patients. Chronic toxicity continues to be a major problem (Burzynski, 2006).

Antineoplastons A10 and AS2-1 are synthetic derivatives of glutamine, isoglutamine, and phenylacetic acid (Burzynski, 2004). A10 is a synthetic formulation consisting of a 4:1 ratio of phenylacetylglutaminate sodium (PG) and phenylacetylisoglutaminate sodium (isoPG). AS2-1 is a synthetic formulation with a 4:1 ratio of phenylacetate sodium (PN) and PG.

In a Phase II study of ANP in patients with an astrocytoma, which was opened for enrollment in 1988, a CR with more than 28 years of progression-free survival (PFS) was documented in a 7-year-old child diagnosed with a large, inoperable OPG (S Burzynski, Kubove \& B Burzynski, 1992; Hawkins \& Friedman, 1992). This patient, who had a short life expectancy and was never treated with RT or chemotherapy, is tumor-free and continues to enjoy good health and a productive life. Additional Phase II studies utilizing ANP have shown objective responses (OR) and improved survival in pediatric high-grade glioma, diffuse intrinsic pontine glioma (DIPG) and low-grade astrocytoma (S Burzynski, Janicki, G Burzynski \& Marszalek, 2014, 2051-2061; S Burzynski, Janicki, G Burzynski \& Marszalek, 2015, 334-344; S Burzynski, Janicki, G Burzynski \& Marszalek, 2014, 565-577; S Burzynski, Janicki, G Burzynski, Marszalek \& Brookman, 2014, 977-988; S Burzynski, Janicki, G Burzynski, Marszalek \& Brookman, 2014, e433-e439, S Burzynski, Janicki, G Burzynski, 2016, 837-850). Such encouraging preliminary data prompted this single-arm Phase II study of ANP in children with an OPG.

\section{Materials and Methods}

\subsection{Patient Population}

This Phase II study accrued patients less than 18 years of age, but more than 6 months of age, who had radiologic evidence of OPG by gadolinium-enhanced magnetic resonance imaging (MRI), which is the diagnostic imaging study of choice (Gutmann \& MacCollin, 2001), performed within 14 days of the start of ANP. The diagnosis of OPG generally does not require a biopsy.

\subsection{Study Design}

Except of diagnosis specific to the protocol, the inclusion and exclusion criteria, as well as reasons for removal from the study, have previously been described (S Burzynski, Janicki, G Burzynski \& Marszalek, 2014, 565-577). Additional patients, including those with a Lansky or Karnofsky score $\leq 50$, were enrolled on a case-by-case basis with an exception granted by the FDA. All patients and/or guardians signed an Informed Consent Document prior to enrollment.

This Phase II study was a single-arm, two-stage, interventional trial of ANP in a high-risk, poor-prognosis study population (Chang et al., 1999). It commenced on June 27, 1996 under IND 43,742 and after approval by the BRI-IRB. The last patient began ANP on 10/13/2004.

The study protocol was amended on occasion, but none of the amendments altered the aim or design of the original study objectives/outcomes or introduced new safety issues.

\subsection{Statistical Considerations}

This was a two-stage study with 20 patients being enrolled in the first stage. If one OR occurred among these patients, up to 20 additional patients could be enrolled (Chang et al., 1999). At the completion of the study, an overall OR rate of $\geq 10 \%$ (i.e., 4 ORs) was considered to warrant further study.

\subsection{Administration of the Medications}

The details of ANP administration have been previously published (Burzynski et al., 2014, 2051-2061; Burzynski et al., 2015; Burzynski et al., 2014, 565-577; Burzynski et al., 2014, 977-988). Escalation of the 
dosage of ANP is based on the results of prior studies carried out to determine patients' tolerance of the infusion of the large volume of intravenous fluids necessary to deliver the higher doses of ANP (Burzynski, 2004). As a safety precaution, escalation of the dosage of ANP will continue through Phase II and Phase III trials.

Medications that were considered necessary for the subjects' welfare and that did not interfere with the evaluation of treatment were given at the discretion of the investigator. The use of corticosteroids was carefully monitored and the smallest dosages compatible with preservation of optimal neurologic functions were utilized.

Treatment with other antineoplastic or immunomodulatory agents was not permitted during the study. Subjects received full supportive care, including transfusions of blood products and antibiotics when appropriate.

\subsection{Evaluation and Follow-up}

OR rates for target lesions were determined using the RANO criteria (Response Assessment in Neuro-Oncology) as assessed by an MRI with gadolinium enhancement. A CR was achieved when disappearance of all disease was sustained for at least four weeks. A PR was achieved with a $\geq 50 \%$ decrease in the sum of the products of the greatest perpendicular diameters of all target lesions, compared to baseline, and sustained for at least four weeks. SD was maintained by a $<50 \%$ decrease in the sum of the products of the greatest perpendicular diameters of all target lesions, compared to baseline, and no progressive disease (PD), sustained for at least four weeks. PD was determined by a $\geq 25 \%$ increase in the sum of the products of the greatest perpendicular diameters of all target lesions compared to the lowest sum previously recorded. Target lesions were contrast-enhancing lesions $\geq 5 \mathrm{~mm}$ in size. (Wen et al., 2010; Weller, Cloughesy, Perry \& Wick, 2013).

Details of the evaluation of overall survival (OS) and adverse drug experiences (ADEs) have been previously described (S Burzynski, Janicki, G Burzynski, 2016, 837-850).

\section{Results}

\subsection{Patient Demographics}

Subject enrollment started June 27, 1996, and continued through October 13, 2004. As of June 5, 2008, all subjects had permanently discontinued ANP due to the achievement of a CR, subject request, $\mathrm{PD}$, or a worsening clinical condition. The 16 patients enrolled had a median age of 4.5 years while. $25 \%$ were female and $75 \%$ were male. All patients were Caucasian.

Patient characteristics are shown in Table 1. 
Table 1. Patient Characteristics

\begin{tabular}{|c|c|c|}
\hline Characteristic & \multicolumn{2}{|l|}{ All Patients $\mathrm{N}=16$} \\
\hline \multicolumn{3}{|l|}{ Age (years) } \\
\hline Median & \multicolumn{2}{|l|}{4.5} \\
\hline Range & \multicolumn{2}{|l|}{$0.6-16.7$} \\
\hline \multicolumn{3}{|l|}{ Sex } \\
\hline Male & \multicolumn{2}{|l|}{$12(75 \%)$} \\
\hline Female & \multicolumn{2}{|l|}{$4(25 \%)$} \\
\hline Ethnicity & \multicolumn{2}{|l|}{ W $16(100 \%)$} \\
\hline \multicolumn{3}{|l|}{ Karnofsky Performance Score } \\
\hline & Treatment Start & Last evaluation on treatment \\
\hline Median & 65 & 85 \\
\hline \multicolumn{3}{|l|}{ Tumor Type } \\
\hline Pilocytic astrocytoma & 5 & 3 solitary, $2 \mathrm{MC}$ \\
\hline Juvenile pilocytic & 2 & 1 solitary, $1 \mathrm{MC}$ \\
\hline Astrocytoma, grade 2 & 3 & 2 solitaries, $1 \mathrm{MF}$ \\
\hline DIG - desmoplastic glioma & 1 & $\mathrm{MC}$ \\
\hline High grade glioma & 1 & Solitary \\
\hline OPG (no biopsy) & 4 & 3 solitary, $1 \mathrm{MC}+\mathrm{L}$ \\
\hline NF-1 associated & 2 & \\
\hline NF-1 unassociated & 14 & \\
\hline \multicolumn{3}{|c|}{ Prior treatment } \\
\hline None or Stereotactic biopsy only & 2 & \\
\hline SU only & 3 & \\
\hline Stereotactic biopsy and $\mathrm{CH}$ & 1 & \\
\hline SU (including excisional biopsy) and $\mathrm{CH}$ & 7 & \\
\hline $\mathrm{CH}$ & 2 & \\
\hline $\mathrm{RT}+\mathrm{CH}$ & 1 & \\
\hline
\end{tabular}

Note. CH-chemotherapy, L- leptomeningeal involvement, MC-multicentric, MF-multifocal, NF-1—neurofibromatosis 1, OPG-optic pathway glioma, RT - radiation therapy, SU - surgery, W—white

\subsection{Treatment}

The trial enrolled 16 study subjects. Three patients received special consideration (one had a Lansky/Karnofsky score of 40 and two had scores of 50). One patient was enrolled despite a lack of tumor contrast enhancement at baseline.

The median dosage of A10 was $8.07 \mathrm{~g} / \mathrm{kg} / \mathrm{d}$ (range 2.53 - $15.83 \mathrm{~g} / \mathrm{kg} / \mathrm{d}$ ) and AS2-1 was $0.39 \mathrm{~g} / \mathrm{kg} / \mathrm{d}$ (range $0.20-0.47 \mathrm{~g} / \mathrm{kg} / \mathrm{d}$ ). The duration of ANP therapy ranged from 4.1 to 210.0 weeks with a median of 38 weeks.

Of the 16 patients enrolled, 2 (12.5\%) achieved a CR, 3 achieved a PR (18.8\%), 5 (31.3\%) maintained SD, while $2(12.5 \%)$ developed PD; the remaining 4 patients did not have follow-up MRIs to be classified. ORs were achieved following a median of 57 weeks of ANP (range, 6 to 177 weeks). The duration of SD ranged from 5.9 to 179.4 months. Characteristics of the patients who achieved a CR or PR are shown in Table 2. 
Table 2. Characteristics of patients who achieved a CR or PR

\begin{tabular}{|c|c|c|c|c|c|c|c|c|c|}
\hline Code & $\begin{array}{c}\text { Age } \\
\text { (years) }\end{array}$ & $\begin{array}{c}\text { Date of initial } \\
\text { diagnosis }\end{array}$ & Histology & NF-1 & Past surgery & $\begin{array}{c}\text { Past } \\
\text { chemotherapy }\end{array}$ & $\begin{array}{c}\text { Past } \\
\text { radiation } \\
\text { therapy }\end{array}$ & Type & $\begin{array}{c}\text { Best } \\
\text { response to } \\
\text { treatment }\end{array}$ \\
\hline 04 & 4 & 02/24/1995 & $\begin{array}{c}\text { Desmoplastic } \\
\text { glioma }\end{array}$ & No & Yes & Yes & No & $\begin{array}{c}\text { BSG/Focal } \\
\text { MC }\end{array}$ & PR \\
\hline 06 & 6 & 10/15/1999 & Pilocytic & No & $\begin{array}{c}\text { Biopsy- } \\
\text { stereotactic }\end{array}$ & No & No & $\begin{array}{c}\text { BSG/Focal } \\
\text { MC }\end{array}$ & CR \\
\hline 07 & 0.75 & 03/13/2000 & $\begin{array}{l}\text { Juvenile } \\
\text { pilocytic }\end{array}$ & No & Yes & Yes & No & MC & PR \\
\hline 09 & 16 & 02/24/1992 & Astrocytoma & Yes & Yes & No & No & Solitary & $\mathrm{CR}$ \\
\hline CE1 & 12 & 07/10/1992 & $\begin{array}{l}\text { High grade } \\
\text { glioma }\end{array}$ & No & Yes & Yes & No & Solitary & PR \\
\hline
\end{tabular}

Note. BSG—brainstem glioma, CE—compassionate exception, CR—complete response, MC—multicentric, NF-1—neurofibromatosis 1, $\mathrm{PR}$ - partial response

Figure 1 presents MRI images of a 6-year old Caucasian female diagnosed with pilocytic astrocytoma of the optic chiasm, optic tract and hypothalamus, without prior treatment, who developed a CR on ANP therapy. ANP started on November 11, 1999 and was discontinued on April 14, 2003. The arrows shown in the images indicate contrast-enhancing target lesions. PET scan of April 5, 2000 confirmed the CR. The patient continues to be tumor-free and has lived a normal life in excess of 15 vears from the start of ANP.
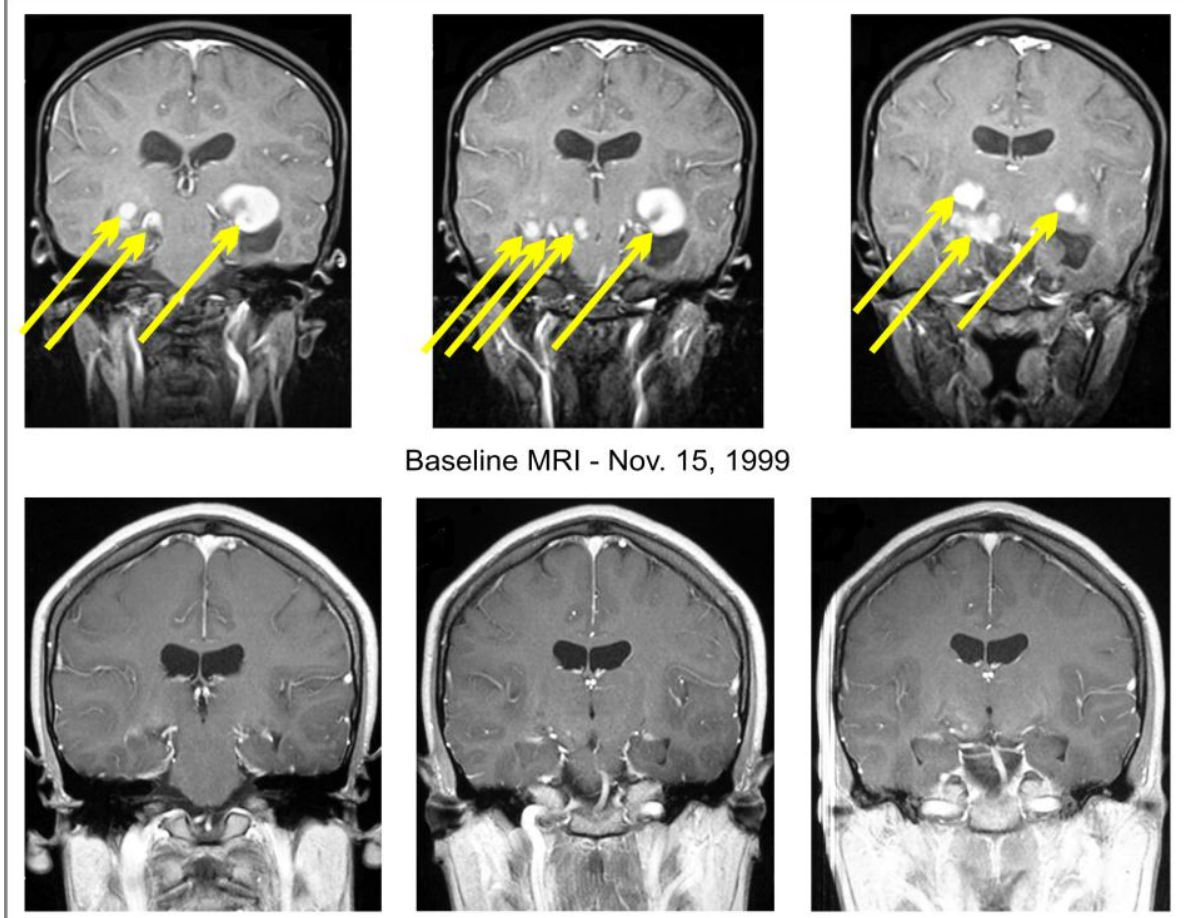

Baseline MRI - Nov. 15, 1999
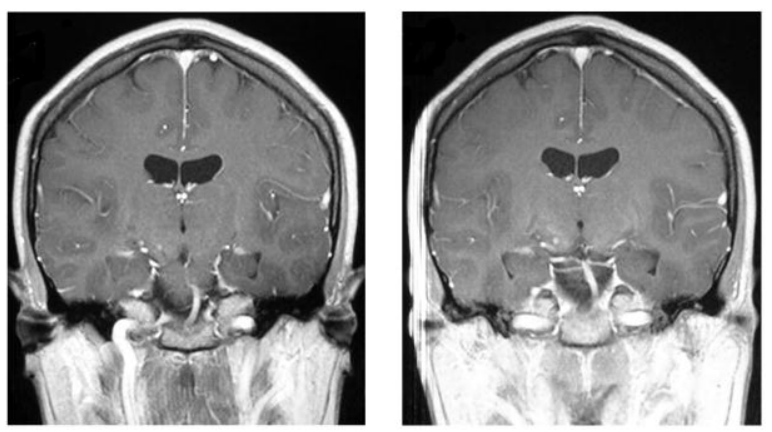

Follow-up MRI - Sep. 25, 2008

Figure 1. Contrast-enhanced MRI images of the brain at baseline (top row of images) and contrast-enhanced MRI images of the brain following ANP therapy demonstrating a CR (bottom row of images)

\subsection{Response and Survival}

It has been suggested that Phase II studies should also determine survival data (Iwamoto \& Lassman, 2015). Therefore, this report includes a survival analysis.

The OS and PFS data for patients living at the time of last contact are presented in Table 3. Over the course of 
treatment, Lansky/Karnofsky score increased in 8 of the 16 patients enrolled, was stable in 5 patients, and decreased in 3 patients.

Table 3. OS and PFS data for patients living at the time of last contact

\begin{tabular}{ccccc}
\hline Code & $\begin{array}{c}\text { Overall survival from diagnosis } \\
\text { in years }\end{array}$ & $\begin{array}{c}\text { Overall survival from treatment } \\
\text { start in years }\end{array}$ & $\begin{array}{c}\text { Progression-free survival in } \\
\text { years }\end{array}$ & $\begin{array}{c}\text { Best Response to } \\
\text { treatment }\end{array}$ \\
\hline 06 & $16.7+$ & $16.65+$ & $16.65+$ & $\mathrm{CR}$ \\
07 & $15.65+$ & $15.5+$ & 3.8 & PR \\
$\mathrm{CE} 1$ & $23.3+$ & $12.6+$ & $12.6+$ & $\mathrm{PR}$ \\
03 & $20.1+$ & $17.7+$ & $17.7+$ & $\mathrm{SD}$ \\
$\mathrm{CE} 2$ & $15.6+$ & $14.9+$ & $14.9+$ & $\mathrm{SD}$ \\
\hline
\end{tabular}

Note. CE-compassionate exception, $\mathrm{CR}$-complete response, $\mathrm{PR}$-partial response, $\mathrm{SD}$-stable disease

Table 4 presents a comparison of our study with other studies reported in the current literature, which utilized chemotherapeutic agents in the treatment of OPG.

Table 4. Comparison of Response of OPG to ANP to that of Other Chemotherapeutic Regimens.

\begin{tabular}{|c|c|c|c|c|c|c|c|c|c|c|c|c|c|}
\hline \multirow{3}{*}{ Author / Treatment } & \multirow{3}{*}{$\begin{array}{l}\text { Number of } \\
\text { patients }\end{array}$} & \multirow{2}{*}{\multicolumn{3}{|c|}{$\begin{array}{c}\text { Efficacy response } \\
(\%)\end{array}$}} & \multicolumn{9}{|c|}{ Kaplan-Meier survival analysis } \\
\hline & & & & & \multicolumn{4}{|c|}{$\begin{array}{c}\text { Progression-free survival } \\
(\%)\end{array}$} & \multicolumn{5}{|c|}{$\begin{array}{c}\text { Overall survival from treatment } \\
\text { start }(\%)\end{array}$} \\
\hline & & $\mathrm{CR}$ & PR & $\mathrm{SD}$ & $\begin{array}{c}2 \\
\mathrm{yrs}\end{array}$ & $\begin{array}{c}3 \\
\mathrm{yrs}\end{array}$ & $\begin{array}{c}4 \\
\mathrm{yrs}\end{array}$ & $\begin{array}{c}5 \\
\mathrm{yrs}\end{array}$ & $\begin{array}{c}2 \\
\text { yrs }\end{array}$ & $\begin{array}{c}3 \\
\mathrm{yrs}\end{array}$ & $\begin{array}{c}5 \\
\text { yrs }\end{array}$ & $\begin{array}{l}10 \\
\text { yrs }\end{array}$ & $\begin{array}{l}17 \\
\mathrm{yrs}\end{array}$ \\
\hline $\begin{array}{l}\text { Gururangan et al 2002, } \\
\text { Carboplatin }\end{array}$ & 30 & 2 & 21 & 51 & & 64 & & & 84 & & & & \\
\hline $\begin{array}{l}\text { Gururangan et al 2007, } \\
\text { Temozolomide }\end{array}$ & 14 & 0 & 0 & 51 & 64 & & 31 & & 71 & 57 & 7 & & \\
\hline $\begin{array}{l}\text { Burzynski et al 2016, } \\
\text { Antineoplastons }\end{array}$ & 16 & 13 & 19 & 31 & 56 & 56 & 49 & 42 & 81 & 74 & 67 & 37 & 37 \\
\hline
\end{tabular}

Note. $\mathrm{CR}$ - complete response, $\mathrm{PR}$ - partial response, $\mathrm{SD}$ - stable disease, yrs-year

Survival analysis revealed a median PFS of 45.4 months and a PFS at 6 months of $75.0 \%$.

The OS at 2 years was $81 \%$, at 4 years it was $67 \%$, at 5 years it was $67 \%$, and at 10 and 15 years it was $37 \%$. The median OS was 78.5 months. The Kaplan-Meier survival curve is shown in Figure 2.

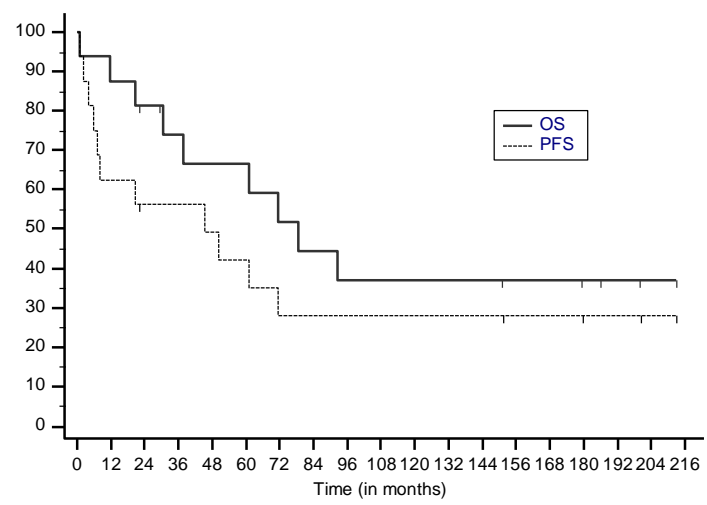

Figure 2. The Kaplan-Meier Survival Curve

Note. OS—overall survival from treatment start, PFS— progression-free survival. 


\subsection{Safety and Adverse Drug Events}

All 16 patients were included in the analysis of toxicity. Patients were initially treated at the Burzynski Clinic. Two or more weeks later, when the patient and/or guardian had been adequately educated in the administration of ANP, they went home. Both at the Burzynski Clinic and at home (via telephone), patients were questioned on a daily basis concerning possible ADEs. After two months, questioning was conducted on a weekly basis. Adverse events were categorized according to CTCAE v 3.0. Table 5 compares the incidence of grade 3 and 4 ADEs in our study to that reported by others. No long-term ADEs to ANP were reported.

Table 5. Incidence of Adverse Drug events, Grades 3 and 4, Reported by Patients During ANP therapy Compared to Those Reported in Studies of OPG Utilizing Chemotherapy.

\begin{tabular}{|c|c|c|c|c|c|}
\hline \multirow{2}{*}{ Adverse drug events } & \multicolumn{2}{|c|}{ Antineoplastons } & \multirow{2}{*}{$\begin{array}{l}\text { Gururangan, et al. } 2002 \\
\text { Carboplatin } \\
\text { Grade } 3 / 4 \\
(\%)\end{array}$} & \multicolumn{2}{|c|}{ Gururangan, et al. 2007 Temozolomide } \\
\hline & Grade $3(\%)$ & Grade $4(\%)$ & & $\begin{array}{c}\text { Grade } 3 \\
(\%)\end{array}$ & $\begin{array}{c}\text { Grade } 4 \\
(\%)\end{array}$ \\
\hline Hypokalemia & & 6 & & & \\
\hline Hypernatremia & & 6 & & & \\
\hline SGOT & 6 & & & & \\
\hline Somnolence & 6 & & & & \\
\hline Thrombocytopenia & & & 58 & & 17 \\
\hline Neutropenia & & & 80 & 3 & 7 \\
\hline Infection & & & 17 & & \\
\hline Allergic reaction & & & 6 & & \\
\hline Hemorrhage & & & & 3 & \\
\hline
\end{tabular}

\section{Discussion}

The successful treatment of OPG, which is not amenable to complete resection, continues to present a clinical challenge. Most of these tumors arise in children younger than five years of age, and their behavior is unpredictable, ranging from spontaneous regression in some patients with neurofibromatosis I to continued neurological impairment leading to disability and death. Most of the reported studies combine patients diagnosed with OPG with low-grade glioma (LGG), and only a small number of clinical investigations deal with OPG exclusively. The studies on LGG have been extensively reviewed in medical literature (Tow et al., 2003; Opocher et al., 2006; Wisoff et al., 1990; Burzynski, 2006; Laithier et al., 2003). Large scale studies have been performed by various groups to develop a comprehensive treatment strategy for all patients with OPG (Laithier et al., 2003; Gnekow et al., 2004). These studies emphasize chemotherapy, but the patients were also treated surgically and with radiation therapy (RT). The main goal was to defer RT by introducing initial combination chemotherapy.

In the study organized by the French Society of Pediatric Oncology, 85 patients were treated with alternating cycles of procarbazine and carboplatin, etoposide and cisplatin, and vincristine and cyclophosphamide, and were followed with RT at progression (Laithier et al., 2003). There were no CRs, but $42 \%$ of patients accomplished PR and $45 \%$ SD. Unfortunately, most of these patients developed disease progression, indicated by PFS of $34 \%$ at five years.

The German Society of Pediatric Oncology and Hematology (GPOH) reported the results of the treatment of 123 patients with vincristine and carboplatin followed by RT at progression (Gnekow et al., 2004). The response rate was CR 2\%, PR 6\%, and 76\% SD. Inevitably, there was progression of the disease, but PFS at five years was reported as $60 \%$.

There are only a small number of reports on Phase II studies in OPG with single chemotherapy regimens and without added RT. The results of two of these studies, utilizing carboplatin and temozolomide, respectively, along with the results of our study, are presented in Table 4 . These three studies enrolled a similar number of patients, but there was a markedly higher CR rate $(12.5 \%)$ in our study compared to a $2 \%$ rate in the carboplatin study and no CRs reported in the temozolomide study (Gururangan, et al., 2002; Gururangan, et al., 2007). There 
were no PRs reported in the temozolomide study while carboplatin treatment provided for a $21 \% \mathrm{PR}$ rate. There was an $18.8 \%$ PR rate in patients utilizing ANP. SD was in the 50\% range for chemotherapy studies and was $31.3 \%$ in patients utilizing ANP.

The carboplatin study did not present survival data beyond two years, but overall survival in the temozolomide trial was $57 \%$ at three years and $7 \%$ at five years, which is comparable to $62 \%$ at three years and $56 \%$ at five years for ANP. OS in excess of 10 and 15 years with ANP (44\%) is substantial. Most patients treated in this study at the Burzynski Clinic had developed tumor recurrence after prior treatments. Fifty percent underwent prior surgical resection and $31 \%$ relapsed after combination chemotherapy before coming to BC. While less disabling than RT, long-term toxicity compromises the survival of children with OPG who are treated with chemotherapy (Burzynski, 2006). Chronic myelosuppression (Perilongo, 2005; De Vita, Carbone, Owens, Gold, Krant \& Edmonson, 1965; Kumar \& Dua, 1987; Calvert, Newell \& Gore, 1992), gonadal dysfunction (Calvert et al., 1992; Rivkees \& Crawford, 1988; Roth, Einhorn \& Greist, 1988; Clayton, Shalet, Price \& Jones, 1989), renal insufficiency (Schilsky, Sherins, Hubbard, Wesley, Young \& DeVita, 1981; Goren, Wright \& Horowitz, 1986; Schacht \& Baldwin, 1978), pulmonary toxicity (Goren et al., 1986; Schacht et al., 1978; Holoye, Jenkins \& Greenberg, 1976; Jones, Moore, Blank \& Castellino, 1972), neurotoxicity (Jones et al., 1972; Shingleton, Bienfang, Albert, Ensminger, Chandler \& Greenberg, 1982; Weiss, Walker \& Wiernik, 1974; Grunberg, Sonka, Stevenson \& Muggia, 1989) including hearing loss (Grunberg et al., 1989; Hansen, Helwig-Larsen \& Trojaborg, 1989; Schaefer, Post, Close \& Wright, 1985) can develop. The use of carmustine and lomustine in the treatment of patients with gliomas is associated with myelofibrosis, secondary myelodysplasia and acute leukemia (Greene, Boice \& Strike, 1985; Cohen, Wiernik \& Walker, 1976).

As indicated by Table 5, treatment with carboplatin and temozolomide is generally less toxic than treatment with carmustine and lomustine, but is associated with a high percentage of Grade 3 and 4 thrombocytopenia and neutropenia. In addition, approximately $17 \%$ of patients in the carboplatin study developed serious infection.

ANP is much better tolerated than cytotoxic chemotherapy. With ANP, there was only a small number of easily reversible Grades 3 and 4 toxicities. There were no chronic ADEs in the ANP study and the quality of survival was very high. A significant number of patients whose tumors went into remission experienced survival in excess of 15 years, with a high quality of life. It is recognized that any single-arm Phase II study is limited by the lack of a control population. However, the patient population in this study was representative of the general population at risk. Efficacy and safety were properly evaluated. Future studies with a larger population of patients can evaluate the efficacy of this regimen in greater detail. It is recognized that OPG comprises a heterogeneous group of brain tumors with over 800 genomic and numerous epigenomic abnormalities (Marko \& Weil, 2012). Currently our group is striving to identify these abnormalities in our patient population and to correlate them with response to ANP.

\section{Conclusion}

This small Phase II study demonstrated an objective response rate of $31.3 \%$ and an overall disease stabilization rate of $62.5 \%$. The long-term survival characteristics are attractive, with $66 \%$ OS at five years and $37 \%$ OS at 15 years. The safety and efficacy of ANP is remarkable with no chronic toxicities and only a small number of easily reversible Grades 3 and 4 toxicities. In future studies it will be important to determine the "genomic signature" of response to ANP. Based on this study, there is now evidence-based research supporting the safety and efficacy of ANP in the treatment of OPG, which should be confirmed by further studies.

\section{Acknowledgments}

The authors express their appreciation to the additional physicians involved in the care of the patients: Robert A. Weaver, M.D., Robert I. Lewy, M.D., Eva Kubove, M.D., Barbara Szymkowski, M.D., and Mohammad Khan, M.D. Preparation of the manuscript was provided by Carolyn Powers and Jenifer Pineda. Ramiro Rivera, M.D. provided assistance in data review. Samuel Beenken, MD provided final review of the manuscript.

\section{References}

Burzynski, S. R. (2004). The present state of antineoplaston research (1). Integrative Cancer Therapy, 3, 47-58. http://dx.doi.org/10.1177/1534735403261964

Burzynski, S. R. (2006). Treatment for astrocytic tumors in children: current and emerging strategies. Pediatric Drugs, 8, 167-78. http://dx.doi.org/10.2165/00148581-200608030-00003

Burzynski, S. R., Janicki, T. J., Burzynski, G. S., \& Marszalek, A. (2014). A phase II study of Antineoplastons A10 and AS2-1 in children with high-grade glioma. Final report (Protocol BT-06), and review of recent trials. Journal of Cancer Therapy, 5, 565-577. http://dx.doi.org/10.4236/jct.2014.56065 
Burzynski, S. R., Janicki, T. J., Burzynski, G. S., \& Marszalek, A. (2014). The response and survival of children with recurrent diffuse intrinsic pontine glioma based on phase II study of Antineoplastons A10 and AS2-1 in patients with brainstem glioma. Child's Nervous System, 30, 2051-2061.

http://dx.doi.org/10.1007/s00381-014-2401-z

Burzynski, S. R., Janicki, T. J., Burzynski, G. S., \& Marszalek, A. (2015). A phase II study of Antineoplastons A10 and AS2-1 in patients with brainstem gliomas. The report on non-diffuse intrinsic pontine glioma (Protocol BT-11). Journal of Cancer Therapy, 6, 334-344. http://dx.doi.org/10.4236/jct.2015.64036

Burzynski, S. R., Janicki, T. J., Burzynski, G. S., Marszalek, A., \& Brookman, S. (2014). Long-term survival (>13 years) in a child with recurrent diffuse pontine gliosarcoma: A case report. Journal of Pediatric Hematology/Oncology, 36, e433-e439. http://dx.doi.org/10.1097/MPH.0000000000000020

Burzynski, S. R., Janicki, T. J., Burzynski, G. S., Marszalek, A., \& Brookman, S. (2014). A phase II study of Antineoplastons A10 and AS2-1 in children with recurrent, refractory or progressive primary brain tumors Final report (Protocol BT-22). Journal of Cancer Therapy, 5, 977-988.

http://dx.doi.org/10.4236/jct.2014.510102

Burzynski, S. R., Janicki, T. J., \& Burzynski, G. S. (2016) A phase II study of Antineoplastons A10 and AS2-1 in children with low-grade astrocytoma - final report (Protocol BT-13). Journal of Cancer Therapy, 7 , 837-850. http://dx.doi.org/10.4236/jct.2016.712083

Burzynski, S. R., Kubove, E., \& Burzynski, B. (1992). Phase II clinical trials of antineoplastons A10 and AS2-1 infusions in astrocytoma. In D. Adam, H. Lode, E. Rubenstein (Eds.), Recent Advances in Chemotherapy: 17th International congress of chemotherapy, Berlin, 1991. Munich, Germany: Futuramed Publishers.

Calvert, A. H., Newell, D. R., \& Gore, M. E. (1992). Future directions with carboplatin: can therapeutic monitoring, high-dose administration, and hematologic support with growth factors expand the spectrum compared with cisplatin? Seminars in Oncology, 19, 155-163.

https://www.ncbi.nlm.nih.gov/pubmed/1411627

Chang, S. M., Kuhn, J. G., Robins, H. I., Schold, S. C., Spence, A. M., Berger, M. S., ... Prados, M. D. (1999). Phase II study of phenylacetate in patients with recurrent malignant glioma: a North American Brain Tumor Consortium report. Journal of Clinical Oncology, 17, 984-990.

http://ascopubs.org/doi/full/10.1200/JCO.1999.17.3.984

Clayton, P. E., Shalet, S. M., Price, D. A., \& Jones, P. H. M. (1989). Ovarian function following chemotherapy for childhood brain tumors. Medical \& Pediatric Oncology, 17, 92-96.

http://dx.doi.org/10.1002/mpo.2950170204

Cohen, R. J., Wiernik, P. H., \& Walker, M. D. (1976). Acute nonlymphocytic leukemia associated with nitrosourea chemotherapy: report of two cases. Cancer Treatment Reports, 60, 1257-1261. https://www.ncbi.nlm.nih.gov/pubmed/1071015

De Vita, V. T., Carbone, P. P., Owens, A. H. Jr, Gold, G. L., Krant, M. J., \& Edmonson, J. (1965). Clinical trials with 1, 3-bis(2-chloroethyl)-1-nitrosourea, NSC-409962. Cancer Research, 25, 1876-1881. http://cancerres.aacrjournals.org/content/25/11_Part_1/1876

Edwards, M. S., \& Cogen, P. H. (1994). Craniospinal neoplasms. In B. O. Berg (Eds.), Child neurology: a clinical manual. $2^{\text {nd }} e d$. (pp. 165-183). Philadelphia, PA: JB Lippincott.

Gnekow, A. K., Kortmann, R. D., Pietsch, T., \& Emser, A. (2004). Low grade chiasmatic-hypothalamic glioma-carboplatin and vincristin chemotherapy effectively defers radiotherapy within a comprehensive treatment strategy -- report from the multicenter treatment study for children and adolescents with a low grade glioma -- HIT-LGG 1996 -- of the Society of Pediatric Oncology and Hematology (GPOH). Klinische Padiatrie, 216, 331-342. http://dx.doi.org/10.1055/s-2004-832355

Goren, M. P., Wright, R. K., \& Horowitz, M. E. (1986). Cumulative renal tubular damage associated with cisplatin nephrotoxicity. Cancer Chemotherapy \& Pharmacology, 18, 69-73. http://dx.doi.org/10.1007/BF00253068

Greene, M. H., Boice, J. D. Jr \& Strike, T. A. (1985). Carmustine as a cause of acute nonlymphocytic leukemia. New England Journal of Medicine, 313, 579. http://dx.doi.org/10.1056/NEJM198508293130911

Grunberg, S. M., Sonka, S., Stevenson, L. L., \& Muggia, F. M. (1989). Progressive paresthesias after cessation of therapy with very high-dose cisplatin. Cancer Chemotherapy \& Pharmacology, 25, 62-64. http://dx.doi.org/10.1007/BF00694340 
Gururangan, S., Cavazos, C. M., Ashley, D., Herndon, J. E., Bruggers, C. S., Moghrabi, A., ... Friedman, H. S. (2002). Phase II study of carboplatin in children with progressive low-grade gliomas. Journal of Clinical Oncology, 20, 2951-2958. http://ascopubs.org/doi/abs/10.1200/JCO.2002.12.008

Gururangan, S., Fisher, M. J., Allen, J. C., Herndon, J. E., Quinn, J. A., Reardon, D. A., ... Friedman, H. S. (2007). Temozolomide in children with progressive low-grade glioma. Neuro-Oncology, 9, 161-168. http://dx.doi.org/10.1215/15228517-2006-030

Gutman, D. H., \& MacCollin, M. M. (2001). Brain tumors associated with neurofibromatosis. In A.H. Kaye \& E.R. Laws (Eds.), Brain tumors: An encyclopedic approach. London, U.K.: Harcourt Publishers Limited.

Hansen, S. W., Helwig-Larsen, S., \& Trojaborg, W. (1989). Long-term neurotoxicity in patients treated with cisplatin, vinblastine, and bleomycin for metastatic germ cell cancer. Journal of Clinical Oncology, 7, 1457-1461. http://ascopubs.org/doi/pdf/10.1200/jco.1989.7.10.1457

Hawkins, M. J., \& Friedman, M. A. (1992). National Cancer Institute's evaluation of unconventional cancer treatments. Journal of the National Cancer Institute, 84, 1699-1702. http://dx.doi.org/10.1093/jnci/84.22.1699

Holoye, P. Y., Jenkins, D. E., \& Greenberg, S. D. (1976). Pulmonary toxicity in long-term administration of BCNU. Cancer Treatment Reports, 60, 1691-1694. https://www.ncbi.nlm.nih.gov/pubmed/1021241

Iwamoto, F. M., \& Lassman, A. B. (2015). Factorial clinical trials: a new approach to phase II neuro-oncology studies. Neuro-Oncology, 17, 174-176. http://dx.doi.org/10.1093/neuonc/nou314

Jahraus, C. D., \& Tarbell, N. J. (2006). Optic pathway gliomas. Pediatric Blood \& Cancer, 46, 586-596. http://dx.doi.org/10.1002/pbc.20655

Janss AJ, Grundy R, Cnaan A, Savino PJ, Packer RJ, Zackai EH, ... Radcliffe J. (1995). Optic pathway and hypothalamic/chiasmatic gliomas in children younger than age 5 years with a 6-year follow-up. Cancer, 75, http://dx.doi.org/10.1002/1097-0142(19950215)75:4<1051::AID-CNCR2820750423>3.0.CO;2-S

Jones, S. E., Moore, N., Blank, N., \& Castellino, R. A. (1972). Hypersensitivity to procarbazine (Matulane) manifested by fever and pleuropulmonary reaction. Cancer, 29, 498-500.

http://onlinelibrary.wiley.com/doi/10.1002/1097-0142(197202)29:2\%3C498::AID-CNCR2820290240\%3E3 $.0 . \mathrm{CO} \% 3 \mathrm{~B} 2-\mathrm{O} / \mathrm{abstract}$

Kumar, L., \& Dua, H. (1987). Cis-platin induced anemia [letter]. New Zealand Medical Journal, 100(817), 81.

Laithier, V., Grill, J., Le Deley, M. C., Ruchoux, M. M., Couanet, D., Doz, F., ... Kalifa, C. (2003). Progression-free survival in children with optic pathway tumors: dependence on age and the quality of the response to chemotherapy--results of the first French prospective study for the French Society of Pediatric Oncology. Journal of Clinical Oncology, 21, 4572-4578. http://dx.doi.org/10.1200/JCO.2003.03.043

Marko, N. F., \& Weil, R. J. (2012). The molecular biology of WHO grade I astrocytomas. Neuro-Oncology, 14, 1424-1431. http://dx.doi.org/10.1093/neuonc/nos257

Opocher, E., Kremer, L. C., Da Dalt, L., van de Wetering, M. D., Viscardi, E., Caron, H. N., \& Perilongo, G. (2006). Prognostic factors for progression of childhood optic pathway glioma: A systematic review. European Journal of Cancer, 42, 1807-1816. http://dx.doi.org/10.1016/j.ejca.2006.02.022

Perilongo, G. (2005). Considerations on the role of chemotherapy and modern radiotherapy in the treatment of childhood low grade glioma. Journal of Neuro-Oncology, 75, 301-307. http://dx.doi.org/10.1007/s11060-005-6754-8

Pollack, I. F., \& Mulvihill, J. J. (1996). Special issues in the management of gliomas in children with neurofibromatosis 1. Journal of Neuro-Oncology, 28, 257-268. http://dx.doi.org/10.1007/BF00250204

Rivkees, S. A., \& Crawford, J. D. (1988). The relationship of gonadal activity and chemotherapy-induced gonadal damage. Journal of the American Medical Association, 259, 2123-2125. http://dx.doi.org/10.1001/jama.1988.03720140043031

Roth, B. J., Einhorn, L. H., \& Greist, A. (1988). Long-term complications of cisplatin-based chemotherapy for testis cancer. Seminars in Oncology, 15, 345-350.

Schacht, R. G. \& Baldwin, D. S. (1978). Chronic interstitial nephritis and renal failure due to nitrosourea (NU) therapy. Kidney International, 14, 661.

Schaefer, S. D., Post, J. D., Close, L. G., \& Wright, C. G. (1985). Ototoxicity of low- and moderate-dose cisplatin. 
Cancer, 56, 1934-1939.

http://onlinelibrary.wiley.com/doi/10.1002/1097-0142(19851015)56:8\%3C1934::AID-CNCR2820560807\% 3E3.0.CO;2-F/abstract

Schilsky, R. L., Sherins, R. J., Hubbard, S. M., Wesley, M. N., Young, R. C., \& DeVita, V. T. (1981). Long-term follow-up of ovarian function in women treated with MOPP chemotherapy for Hodgkin's disease. American Journal of Medicine, 71, 552-556. http://dx.doi.org/10.1016/0002-9343(81)90202-3

Shingleton, B. J., Bienfang, D. C., Albert, D. M., Ensminger, W. D., Chandler, W. F., \& Greenberg, H. S. (1982). Ocular toxicity associated with high-dose carmustine. Archives of Ophthalmology, 100, 1766-1772. http://dx.doi.org/10.1001/archopht.1982.01030040746007

Thiagalingam, S., Flaherty, M., Billson, F., \& North, K. (2004). Neurofibromatosis type 1 and optic pathway gliomas: follow-up of 54 patients. Ophthalmology, 111, 568-577. http://dx.doi.org/10.1016/j.ophtha.2003.06.008

Tow, S. L., Chandela, S., Miller, N. R., \& Avellino, A. M. (2003). Long-term outcome in children with gliomas of the anterior visual pathway. Pediatric Neurology, 28, 262-270. http://dx.doi.org/10.1016/S0887-8994(02)00628-8

Weiss, H. D., Walker, M. D., \& Wiernik, P. H. (1974). Neurotoxicity of commonly used antineoplastic agents (second of two parts). New England Journal of Medicine, 291, 127-133. http://dx.doi.org/10.1056/NEJM197407182910305

Weller, M., Cloughesy, T., Perry, J. R., \& Wick, W. (2013). Standards of care for treatment of recurrent glioblastoma-are we there yet? Neuro-Oncology, 15, 4-27. http://dx.doi.org/10.1093/neuonc/nos273

Wen, P. Y., Macdonald, D. R., Reardon, D. A., Cloughesy, T. F., Sorensen, A. G., Galanis, E., ... Chang, S. M. (2010). Updated response assessment criteria for high-grade gliomas: Response assessment in Neuro-oncology Working Group. Journal of Clinical Oncology, 28, 1963-1972. http://dx.doi.org/10.1200/JCO.2009.26.3541

Wisoff, J. H., Abbott, R. \& Epstein, F. (1990). Surgical management of exophytic chiasmatic-hypothalamic tumors of childhood. Journal of Neurosurgery, 73, 661-667. http://thejns.org/doi/full/10.3171/jns.1990.73.5.0661

\section{Copyrights}

Copyright for this article is retained by the author(s), with first publication rights granted to the journal.

This is an open-access article distributed under the terms and conditions of the Creative Commons Attribution license (http://creativecommons.org/licenses/by/4.0/). 